EVOLUTIONARY DEVELOPMENTAL BIOLOGY

\section{More than one way to a central nervous system}

\begin{abstract}
Have the molecular mechanisms that are linked to the developmental organization of centralized nervous systems evolved once or multiple times? Evidence from nine animal species points to the latter.
\end{abstract}

\section{CAROLINE B. ALBERTIN \\ \& CLIFTON W. RAGSDALE}

A nimal nervous systems come in many shapes and sizes, ranging from a handful of neurons to large, complex brains. A key question has been whether the centralized nervous systems found in many bilaterally symmetrical animals (bilaterians), which include vertebrates and insects, share a common evolutionary origin, or evolved more than once. At a superficial level, both flies and vertebrates boast a brain connected to a single nerve cord that extends into the trunk. In addition, molecular data indicate that key regulatory genes are deployed similarly during nervous-system development in vertebrates, flies ${ }^{1}$ and another bilaterian, a segmented worm (an annelid) ${ }^{2}$. These similarities have been interpreted as evidence for evolutionary conservation of an ancient bilaterian developmental program for centralized nervous systems. But in a paper online in Nature, Martín-Durán and colleagues ${ }^{3}$ provide evidence for the independent evolution of such nervous systems.

The evolutionary steps between a nerve net and the elaborate centralized nervous systems of bilaterians have been an area of active interest for more than a century ${ }^{4}$. In the mid-1980s, our ability to study this process received a boost, thanks to the discovery of a large family of genes that encode transcription factors containing a DNA-binding homeobox domain ${ }^{5}$. It emerged that members of this homeoboxgene family, including the Hox complex, are expressed in the same order along the headto-tail (anterior-posterior) axis during development in many distantly related bilaterians, including flies and vertebrates ${ }^{5}$. It was later shown that a signalling pathway governed by genes that encode bone morphogenetic proteins (BMPs) is needed to establish the dorsal-ventral (back-to-belly) body axis in diverse bilaterians $^{6}$.

Given these insights, it was not surprising to find that a suite of homeobox genes is also expressed in strikingly similar patterns along the dorsal-ventral axis of the developing nervous systems of vertebrates and fruit flies ${ }^{1}$. Along this axis, staggered homeobox-gene expression correlates with the development of specific neuron types in different regions. The discovery that these genes are also expressed along the dorsal-ventral nervous-system axis in Platynereis dumerilii (an annelid distantly related to flies and vertebrates) was seen as evidence that bilaterian nerve cords are evolutionarily conserved ${ }^{2}$.

Advances in phylogenetic methods for analysing evolutionary relationships, coupled with broader sampling across the evolutionary tree, have altered our understanding of animal relationships. In 2016, a phylogenetic analysis identified an assemblage of small, bilaterally symmetrical, simple worms, collectively referred to as xenacoelomorphs, as the sister group to the rest of the bilaterians (nephrozoans) $)^{7}$. Because xenacoelomorphs are the closest living relatives to the nephrozoans (Fig. 1), comparisons between these two groups can help researchers to infer traits present in the last common ancestor of all bilaterians.

Xenacoelomorphs display diverse nervous-system arrangements. Some have only a nerve net, like the closest relatives of bilaterians, the cnidarians (jellyfish and sea anemones). Others also have one or more nerve cords that are located dorsally, ventrally ventral axis. Martín-Durán and colleagues investigated the expression of patterning genes in four xenacoelomorph species. They found that, although the expression of BMP and anterior-posterior homeobox genes in these species was consistent with patterns seen in other bilaterians, the expression of the dorsal-ventral homeobox genes in the nervous system was not.

Martín-Durán et al. next investigated dorsal-ventral patterning in the nephrozoans. For this work, they extended their analysis of dorsal-ventral homeobox genes to five species within the Spiralia, a large but or at multiple positions along the dorsal-

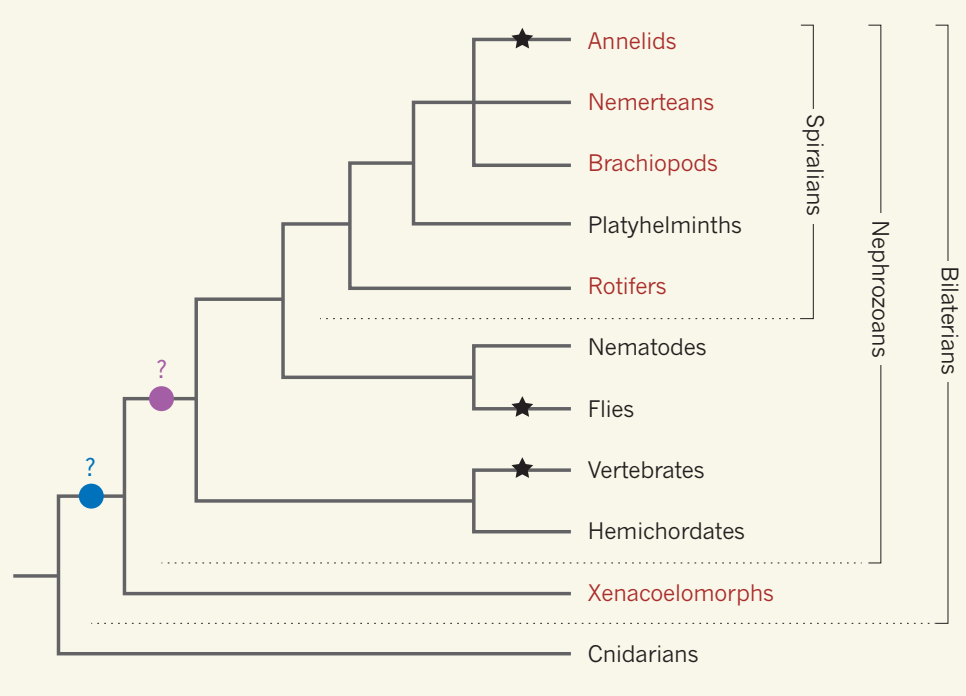

Figure 1 | Evolution of animal nervous systems. The bilaterians (animals that show bilateral symmetry) consist of nephrozoans and a sister group, xenacoelomorphs. Many nephrozoans and xenacoelomorphs have centralized nervous systems, unlike their closest relatives, cnidarians, which feature a simple nerve net. A suite of homeobox genes is expressed (stars) along the back-to-belly axis of the central nervous systems of vertebrates, flies and a segmented worm (an annelid), and it has been posited that this is an evolutionarily conserved gene-expression pattern guiding the development of a centralized nervous system that originated from a common bilaterian (blue circle) or nephrozoan (purple circle) ancestor. However, Martín-Durán et al. ${ }^{3}$ did not find this pattern in nine bilaterian species - five spiralians and four xenacoelomorphs (red text). Their data strengthen the case that the developmental and morphological similarities between bilaterian centralized nervous systems are the result of independent evolutionary events that converged on similar outcomes. 
little-studied bilaterian group that includes annelids, flatworms and molluscs (Fig. 1). The researchers found that the anticipated dorsalventral homeobox pattern was rarely observed, even in part, in the nervous systems of these species, including in an annelid closely related to $P$. dumerilii. These results suggest that even closely related species that have similar nervous-system architectures can deploy ancient genes very differently.

Previous studies in acorn worms ${ }^{8}$ (hemichordates) and flatworms ${ }^{9}$ found no dorsal-ventral homeobox-gene expression in their trunk nervous systems. This absence was previously interpreted as a secondary loss of an ancestral neural patterning system. But in light of Martín-Durán and colleagues' data, this condition could, in fact, reflect the ancestral nephrozoan state. It now seems that the 'typical' dorsoventral gene network was not deployed in the nervous system of the last common ancestor of bilaterians or nephrozoans. Rather, the developmental mechanisms that pattern the neural cords in mice, flies and P. dumerilii might have evolved convergently.

Martín-Durán and colleagues' work paints a complex and nuanced picture of nervous-system evolution. Their data raise the possibility of multiple origins of animal nerve cords, and suggest that a suite of genes that pattern the dorsal-ventral axis has been repeatedly co-opted into nervous-system development. Indeed, the authors show that the relationship between an animal's morphology and the expression of particular developmental genes might not always be tightly linked. These insights raise exciting questions about the mechanisms of evolutionary change that underlie the development of morphological diversity, including why convergently evolved nervous systems sometimes use highly conserved suites of genes, and what developmental constraints govern variations in these mechanisms across animals.

A frequent criticism of the study of key model organisms such as fruit flies, mice and nematode worms is that these species are highly derived - that is, they contain many traits unique to them - and thus are unlike any distant ancestor. But all living species are highly derived, being shaped by natural and sexual selection on evolutionary timescales to maintain adaptation to varying ecological niches. What Martín-Durán and co-workers have highlighted is not that these model organisms are inappropriate 'reference species" ${ }^{10}$.
Rather, they demonstrate the importance both of developing reference species for multiple groups within a robust phylogenetic framework, and of consistently examining close relatives of the reference species before drawing conclusions about the evolutionary history of shared features.

Caroline B. Albertin and Clifton W. Ragsdale are in the Department of Neurobiology,

The University of Chicago, Chicago,

Illinois 60637, USA.

e-mails: calbertin@uchicago.edu;

cragsdale@uchicago.edu

1. Cornell, R. A. \& Von Ohlen, T. Curr. Opin. Neurobiol. 10, 63-71 (2000).

2. Denes, A. S. et al. Cell 129, 277-288 (2007).

3. Martín-Durán, J. M. et al. Nature http:dx.doi. org/10.1038/nature25030 (2017).

4. Dohrn, A. \& Ghiselin, M. T. Hist. Phil. Life Sci. 16, 3-96 (1994).

5. McGinnis, W. \& Krumlauf, R. Cell 68, 283-302 (1992).

6. De Robertis, E. M. \& Sasai, Y. Nature $\mathbf{3 8 0 , 3 7 - 4 0}$ (1996).

7. Cannon, J. T. et al. Nature 530, 89-93 (2016).

8. Lowe, C. J. et al. PLoS Biol. 4, e291 (2006).

9. Scimone, M. L., Kravarik, K. M., Lapan, S. W. \& Reddien, P. W. Stem Cell Rep. 3, 339-352 (2014).

10.Striedter, G. F. et al. Brain Behav. Evol. 83, 1-8 (2014). 